\title{
On Exploring the Various Challenges and Success Criteria towards the E-waste Management in Developing Countries and Studying the Possible Interrelationships amongst them
}

\author{
Viraj Voditel \\ Techture Structures \\ Private Limited \\ Nagpur, India
}

\author{
Remica Aggarwal \\ Recventures Education \\ Services Private Limited, \\ Delhi, India
}

\author{
Lakshay Aggarwal \\ Sociobuddy Technologies \\ Private Limited \\ Delhi, India
}

\author{
V. K. Aggarwal \\ Recventures Education \\ Services Private Limited, \\ Delhi, India
}

\begin{abstract}
Economies of $e$-waste hinges on many factors. Proposed research work explores the various challenges and success factors towards the e-waste management . Thereafter it explores the relationship amongst them using ISM methodology. The research is a mix of exploratory and application based.
\end{abstract}

\section{Keywords}

E-waste ; ISM methodology ; Hazardous ; Non-hazardous ; $e$ waste management

\section{INTRODUCTION}

A typical developing country like India has been facing the issue of safe and efficient handling of $e$-waste ever since the revolution in Information Technology. The availability and affordability of a whole range of electronic equipment coupled with innovations and changing trends have led to rapid rate of obsolescence. India is the fifth biggest generator of e-waste in world (United Nations University, 2014). Currently around 15 lakh metric tons (MT) per annum of $e$ waste is generated and its compound annual growth rate is about 25\% [1]. Almost $60 \%$ of e-waste is a mix of large and small electrical and electronic equipment used in homes and businesses. Indian Ministry of Environment, Forest and Climate Change, notified $e$-waste rules which came into force with effect from $1^{\text {st }}$ May 2012. Implementation of EPR (Extended Producers Responsibility) and mandatory registration of $e$-waste recycling firms with Pollution Control Boards are the key salient features of e-waste rules. Bangalore (Karnataka , India $)^{1}$ has over 1,200 overseas and domestic electronic industries, which pushes in the list of cities facing the menace of $e$-waste hazard. Bangalore generates nearly 86000 MT of $e$-waste per annum and has 31 registered $e$ waste recycling/dismantling firms of which only 3 are actively involved in recycling. The paper is arranged as follows . Section 2 presents the literature review on the various barriers or challenges faced in $e$-waste management in India. Thereafter, in section 3, ISM methodology is explained with its application to case problem in section 4. Finally, the research work is concluded with implications and future directions in section 5 .

\section{E-WASTE MANAGEMENT IN INDIA}

\subsection{E-waste issues [3-19]}

1. Legal issues, lack of research, lack of infrastructure [3Ls]:These barriers are also highlighted as major barriers in ISM models developed for green supply chain [2].
2. Poor information and awareness on $\boldsymbol{e}$-waste generation rates $[\mathbf{P I R}]:$ In addition to domestic generation, $e$-waste is also imported from developed economies, often illegally. There is little understanding of the nature and amount of $e$ waste that gets imported into the country. Also failure in implementation of the regulation can be attributed to low levels of awareness among consumers and waste generators. There is very little understanding about the stakeholders and their role in $e$-waste management.

3. Environmentally unsustainable informal sector practices [EUIS] : The lack of awareness regarding e-waste and costs of returning the end-of-life equipment to formal collection centres are reducing the willingness of household and institutional consumers to return their waste to formal sector despite its growth in formal dismantling and recycling sector .

4. Environmental and health hazards [EHH] : The informal sector's waste management practices pose serious environmental and health hazards to the workers themselves as well as the larger public.

5. Significant capital expenditures [SCE] : Employing effective recycling technologies for $e$-waste may require significant upfront capital expenditures, which may not be justified for private entities in the absence of certainty around sourcing of enough quantities of $e$-waste.

6. Inadequate regulatory design and enforcement [IRDE]: In the 2012 regulations, the mandatory take back system for producers, without accompanying collection targets, provided no incentives to take responsibility and thus induced little improvements in $e$-waste management practices.

7. Informal sector access to waste and flexibility [AWF] : Informal sector's structure and its inherent flexibility and its ability to access waste from both individuals and business is extremely critical as it captures most waste that is generated. It poses a serious challenge to the formal sector and its ability to compete making implementation of $e$-waste regulations extremely challenging.

8. Hazardous refining processes [HRP]: The processes involved in material refining is perhaps the biggest challenge to environment on counts of resource efficiency and absence of appropriate technology. It is also well established that collection and aggregation does not compromise environmental integrity.

9. Illegal transportation of goods [ITG] : On one hand, the tax regime creates a situation where collection and transportation of waste by any individual or group is being 
legitimized and accepted by state. On the other hand, the illegality of transporting these goods is explicit in the $e$-waste rules which are completely overlooked by charging GST and thereby granting legitimacy.

10. Financing gap [FG] : Financing gap exists between informal and formal systems. Individual consumers in India generating $e$-waste have become accustomed to being paid for their $e$-waste when collected by the informal sector. Since informal recyclers externalize health, safety and environmental costs, they are often able to offer a better price for this $e$-waste than formalized recyclers that apply environmental and safety standards.

11. Lack of recognition [LoR] : Actions, interventions and initiatives undertaken by variety of NGO's such as Toxics Link, Chintan, social enterprises SAAHAS, informal sector associations and unions like SWaCH, HRA, SEWA etc. and international agencies like GIZ and EU often demotivate the informal sector workers who have invested in shifting to formal setups [11].

12. Cherry picking $(\mathbf{C P}]$ : The $e$-waste rules cover both IT waste such as computers, mobiles, phones, and so on, and consumer electronics such as televisions, refrigerators, washing machines, air conditioners and lamps. Unsurprisingly, cherry picking - whereby only the positive value fractions are recycled-is rife. Negative value fractions, such as Cathode Ray Tube (CRT) TVs or lamps, are not found attractive, and therefore not accepted by many recyclers.

13. Mind-set of minimal compliance (MSMC): From large multinational original equipment manufacturers (OEMs) to small importers of EEE, companies are focused on keeping compliance costs for $e$-waste management at the minimum possible, and willing to cut corners where possible.

14. Absence of financing for monitoring and control (AoF): Poor recycling and recovery processes mean lower revenues from the materials, creating larger financing gaps. Recyclers want monitoring to ensure more producers are financing formal recycling and there is a crackdown on informal recycling; producers want monitoring to ensure recyclers meet standards and are not paper trading.

15. Poor logistics complicated by geographic realities (PL) : Poor logistic networks add to the costs of aggregation and storage. These coupled with the fact that the country's recycling capacity is concentrated in a few urban areas, make transportation of e-waste expensive.

16. Lack of adequate metal recycling practice (LARP): Another significant challenge to more responsible $e$-waste management results from India's lack of adequate metals recycling capacity. Most formal recyclers in India are dismantlers, with few formal facilities capable of extracting precious metals.

17. Consumer Incentives and Awareness(CIA): Bulk or institutional consumers are largely unaware of their legal liability for $e$-waste management and filing $e$-waste returns. Even those who are aware, for example, the government departments, are unwilling to provide PROs with access to their e-waste as the concept of PROs is still not mainstream.

18. Unreasonable expectations (UE) : The other bulk consumers, such as corporate offices, banks and educational institutions, have unreasonable expectations on the financial returns for responsible recycling of their e-waste. For example, bulk consumers usually expect ₹1500-2000/piece for a laptop when the real price that can be offered if the product is responsibly recycled is around ₹200-300.

19. Malpractice (MP) : Instances of malpractices includes systemic leakages from authorized recyclers due to the focus on collection of waste that gives higher returns to the informal sector aggregators/recyclers. Another instance of a malpractice reported is the issuance of certificates of recycling for materials that have not even been sent to recycling by the recyclers. There are also instances of multiple accounting of e-waste [30].

20. Compliance mind-set (CMS) : In the absence of other drivers such as consumer demand, environmental leadership and resource efficiency, businesses tend to design their response to keep compliance costs as low as possible. The lack of sufficient regulatory capacity at central and state levels, in terms of manpower, financial and non-financial resources as well as lack of established body of knowledge and business models further limit businesses' response to regulations on $e$-waste management.

\subsection{Success factors behind creating a robust $e$-waste management system}

2.2.1. Working towards cross- sector partnerships [CSP] : As informal sector as a critical stakeholder in any future $e$ waste regime, addressing the problem of informal sector $e$ waste practices could be constituted under the Ministry of Environment, Forest and Climate Change (MoEFCC) at the central level and under the State Departments of Environment at the state level.

2.2.2. Generation of revenue [GoR] : Economic instruments such as advanced recycling fee (ARF) or advanced disposal fee (ADF) on every unit of the product sold in the market would relieve the producers of the physical responsibility of collection and the revenues generated could be used to develop markets for the end-of-the-life products. Some examples include (a) subsidize consumers to deposit their $e$ waste at designated centres, (b) directly fund recyclers or PROs and (c) assist informal sector workers in training or skill development or provide greater social security net to the workers.

2.2.3. Regulatory enforcement [RE]: Regulatory actions such as authorizations and their conditions, data on inspections of registered facilities and compliance status of inspected facilities should all be made publicly available for scrutiny. Unequal access to business information and the changing nature of information flows are important factors. Further it has been seen that women are more likely to work in lower-paid, more hazardous activities associated with $e$ waste dismantling and recycling, or be paid less for the same work as men.

2.2.4. E-waste imports for reuse and recycling [IRR] : Under the existing regulations, $\boldsymbol{e}$-waste is not allowed to be imported for final disposal but can be imported for reuse and recycling. In the absence of adequate infrastructure in the country for recycling banning all kinds of imports should be seriously considered.

2.2.5. Public awareness [PA] : The current e-waste regulations require the producers to provide, on their websites, information on the impacts of $e$-waste, appropriate disposal practices and such other issues. They are also required to run awareness campaigns at regular intervals. Many producers have already provided information on their websites, but evidence shows that the overall awareness levels, even among bulk consumers, remain low. 
2.2.6. Advertising and Information campaigns[AIC] : Stricter guidelines/regulations to the producers on the frequency and mode of the awareness campaigns might improve the situation. Alternatively, the producers should be mandated to run these campaigns through grassroots-level organizations working in the area of e-waste. Also, integrating $e$-waste awareness campaigns initiatives with other waste streams such as batteries and municipal solid waste could be a success criteria. The awareness efforts should be geared towards not only achieving safe handling of e-waste but also reducing consumption of electronic products in the long run. In addition, the public awareness generation initiatives should be based on partnerships and collaboration among various stakeholders.

2.2.7. Better funding mechanisms [BFM] : A clearly communicated roll-out plan, notified well in advance, will help the producers make the necessary strategic and budgetary plans. The scale and diversity of skills and knowledge required for e-waste management necessitates building capacity at all levels and of all stakeholders, from policymakers and regulators at the federal and state levels, managers at PROs and other compliance services providers, dismantlers, recyclers as well as entrepreneurs, investors, researchers and academics. The investment in skills and capacity has a direct bearing on the overall costs of the system. This would mean establishing funding mechanisms and structured programmes that build capacity.

2.2.8. Technology-enabled monitoring mechanism [TEM]: All stakeholders agree that enforcement action by the government is essential. One of the first steps should be to introduce an online registry system for reporting by producers, PROs and recyclers. The system should be able to identify and raise red flags for regulators to have more intelligence-based monitoring.

\section{INTERPRETIVE STRUCTURAL MODELLING METHODOLOGY}

Interpretive structural modelling methodology or ISM [1974] is a known technique to map the relationships amongst the relevant elements as per decision maker's problems in a hierarchical manner. Starting with the identification of elements, it proceeds with establishing the contextual relationships between elements (by examining them in pairs ) and move on towards developing the structural selfinteraction (SSIM) matrix using VAXO [1974] and then initial reachability matrix and final reachability matrix and rearranging the elements in topological order using the level partition matrices. A Mic-Mac analysis is performed afterwards which categorize the variables as per the driving and dependence power in to autonomous, dependent, driver and linkage category. Finally, a diagraph can be obtained.

\section{DEVELOPMENT OF ISM MODEL : CASE EXAMPLE}

\subsection{Success factors behind $e$-waste management}

Around 20 challenges viz. Legal issues, lack of research, lack of infrastructure [3Ls]; Poor information and awareness on $e$ waste generation rates [PIR] ; Environmentally unsustainable informal sector practices [EUIS] ; Environmental health hazards [EHH] ; Significant capital expenditures [SCE]; Inadequate regulatory design and enforcement [IRDE]; Informal sector access to waste and flexibility [AWF]; Hazardous refining processes [HRP]; Illegal transportation of goods [ITG]; Financing gap [ FG]; Lack of recognition [LoR]; Cherry picking (CP] ; Mind-set of minimal compliance (MSMC); Absence of financing for monitoring and control (AoF) ; Poor logistics complicated by geographic realities (PL); Lack of adequate metal recycling practice (LARP); Consumer Incentives and Awareness (CIA); Unreasonable expectations (UE); Malpractice (MP); Compliance mind-set (CMS). Similarly, 08 success factors viz. Working towards cross- sector partnerships [CSP]; Generation of revenue [GoR]; Regulatory enforcement [RE]; E-waste imports for reuse and recycling [IRR]; Public awareness [ PA]; Advertising and Information campaigns[AIC] ; Better funding mechanisms [BFM]; Technology-enabled monitoring mechanism [TEM] described above in section 2 have been studied for possible interrelationships amongst them .

4.1.1 Construction of Structural self- interaction Matrix (SSIM)

This matrix gives the pair-wise relationship between two variables i.e. $\mathrm{i}$ and $\mathrm{j}$ based on VAXO. SSIM has been presented below in Fig 1.

4.1.2 Construction of Initial Reachability Matrix and final reachability matrix

The SSIM has been converted in to a binary matrix called the initial reachability matrix shown in fig. 2 by substituting V, A, $\mathrm{X}, \mathrm{O}$ by 1 or 0 as per the case. After incorporating the transitivity, the final reachability matrix is shown below in the Fig 3.

\subsection{Barriers to e-waste management}

08 success factors viz. Working towards cross- sector partnerships [CSP]; Generation of revenue [GoR]; Regulatory enforcement [RE]; E-waste imports for reuse and recycling [IRR]; Public awareness [PA]; Advertising and Information campaigns $[\mathrm{AIC}]$; Better funding mechanisms [BFM]; Technology-enabled monitoring mechanism [TEM] described above in section 2 have been studied for possible interrelationships amongst them .

4.2.1 Construction of Structural self- interaction Matrix (SSIM)

This matrix gives the pair-wise relationship between two variables i.e. $\mathrm{i}$ and $\mathrm{j}$ based on VAXO. SSIM has been presented below in Fig 4.

4.2.2 Construction of Initial Reachability Matrix and final reachability matrix

The SSIM has been converted in to a binary matrix called the initial reachability matrix shown in fig. 5 by substituting $\mathrm{V}, \mathrm{A}$, $\mathrm{X}, \mathrm{O}$ by 1 or 0 as per the case. After incorporating the transitivity, the final reachability matrix is shown below in the Fig 6. 


\begin{tabular}{|c|c|c|c|c|c|c|c|c|c|c|c|c|c|c|c|c|c|c|c|c|c|}
\hline $\begin{array}{l}\text { S. } \\
\text { N } \\
\text { o. }\end{array}$ & $\begin{array}{l}\text { Barri } \\
\text { ers }\end{array}$ & 1 & 2 & 3 & 4 & 5 & 6 & 7 & 8 & 9 & $\begin{array}{l}1 \\
0\end{array}$ & 11 & $\begin{array}{l}1 \\
2\end{array}$ & 13 & 14 & $\begin{array}{l}1 \\
5\end{array}$ & 16 & 17 & $\begin{array}{l}1 \\
8\end{array}$ & 19 & 20 \\
\hline & & $\begin{array}{c}3 \\
\text { Ls }\end{array}$ & $\begin{array}{l}\text { PI } \\
\text { R }\end{array}$ & $\begin{array}{l}\text { EU } \\
\text { IS }\end{array}$ & $\begin{array}{c}\text { EH } \\
H\end{array}$ & $\begin{array}{c}\mathrm{SC} \\
\mathrm{E}\end{array}$ & $\begin{array}{c}\text { IRD } \\
\text { E }\end{array}$ & $\begin{array}{c}\text { A } \\
\text { WF }\end{array}$ & $\begin{array}{c}\mathrm{HR} \\
\mathrm{P}\end{array}$ & $\begin{array}{l}\text { IT } \\
\text { G }\end{array}$ & $\begin{array}{l}F \\
G\end{array}$ & $\begin{array}{c}\text { Lo } \\
\text { R }\end{array}$ & $\begin{array}{l}\mathrm{C} \\
\mathrm{P}\end{array}$ & $\begin{array}{l}\text { MS } \\
\text { MC }\end{array}$ & $\begin{array}{l}\text { Ao } \\
F\end{array}$ & $\begin{array}{l}\mathrm{P} \\
\mathrm{L}\end{array}$ & $\begin{array}{l}\text { LA } \\
\text { RP }\end{array}$ & $\begin{array}{c}\text { CI } \\
\text { A }\end{array}$ & $\begin{array}{l}\mathrm{U} \\
\mathrm{E}\end{array}$ & $\begin{array}{l}\mathrm{M} \\
\mathrm{P}\end{array}$ & $\begin{array}{c}\mathrm{CM} \\
\mathrm{S}\end{array}$ \\
\hline 1 & 3Ls & & V & $\mathrm{V}$ & $\mathrm{V}$ & $\mathrm{V}$ & V & $\mathrm{V}$ & V & $\mathrm{V}$ & $\mathrm{V}$ & $\mathrm{V}$ & $\mathrm{V}$ & $\mathrm{V}$ & $\mathrm{V}$ & V & $\mathrm{V}$ & $\mathrm{V}$ & V & $\mathrm{V}$ & $\mathrm{V}$ \\
\hline 2 & PIR & & & $\mathrm{V}$ & $\mathrm{V}$ & $\mathrm{V}$ & $\mathrm{V}$ & $\mathrm{V}$ & $\mathrm{V}$ & $\mathrm{V}$ & $\mathrm{V}$ & $\mathrm{V}$ & $\mathrm{V}$ & $\mathrm{V}$ & $\mathrm{V}$ & $\mathrm{V}$ & $\mathrm{V}$ & $\mathrm{V}$ & $\mathrm{V}$ & $\mathrm{V}$ & $\mathrm{V}$ \\
\hline 3 & EUIS & & & & $\mathrm{V}$ & $\mathrm{V}$ & $\mathrm{V}$ & $\mathrm{V}$ & $\mathrm{V}$ & $\mathrm{V}$ & $\mathrm{V}$ & $\mathrm{V}$ & $\mathrm{V}$ & $\mathrm{V}$ & $\mathrm{V}$ & $\mathrm{V}$ & $\mathrm{V}$ & $\mathrm{V}$ & $\mathrm{V}$ & $\mathrm{V}$ & $\mathrm{V}$ \\
\hline 4 & EHH & & & & & $\mathrm{V}$ & A & $\mathrm{A}$ & $\mathrm{A}$ & $\mathrm{A}$ & A & $\mathrm{A}$ & $\mathrm{A}$ & $\bar{A}$ & $\mathrm{~A}$ & $\overline{\mathrm{A}}$ & $\mathrm{A}$ & $\mathrm{A}$ & $\mathrm{A}$ & $\mathrm{A}$ & $\mathrm{A}$ \\
\hline 5 & SCE & & & & & & $\bar{A}$ & $\mathrm{~A}$ & $\mathrm{~A}$ & $\mathrm{~A}$ & $\mathrm{~A}$ & $\mathrm{~A}$ & $\bar{A}$ & $\mathrm{~A}$ & $\mathrm{~A}$ & $\bar{A}$ & $\mathrm{~A}$ & $\mathrm{~A}$ & $\mathrm{~A}$ & $\mathrm{~A}$ & $\mathrm{~A}$ \\
\hline 6 & IRDE & & & & & & & $\mathrm{V}$ & $\mathrm{V}$ & $\mathrm{V}$ & $\mathrm{V}$ & $\mathrm{V}$ & $\mathrm{V}$ & $\mathrm{V}$ & $\mathrm{V}$ & $\mathrm{V}$ & $\mathrm{V}$ & $\mathrm{V}$ & $\mathrm{V}$ & $\mathrm{V}$ & $\mathrm{V}$ \\
\hline 7 & AWF & & & & & & & & $\mathrm{V}$ & $\bar{V}$ & $\mathrm{~V}$ & $\mathrm{~V}$ & $\mathrm{~V}$ & $\mathrm{~V}$ & $\mathrm{~V}$ & $\mathrm{~V}$ & $\mathrm{~V}$ & $\mathrm{~V}$ & $\mathrm{~V}$ & $\mathrm{~V}$ & $\mathrm{~V}$ \\
\hline 8 & HRP & & & & & & & & & $\bar{A}$ & $\bar{A}$ & $\mathrm{~A}$ & $\bar{A}$ & $\mathrm{~A}$ & $\mathrm{~A}$ & A & $\mathrm{A}$ & $\mathrm{A}$ & $\bar{A}$ & $\mathrm{~A}$ & $\mathrm{~A}$ \\
\hline 9 & ITG & & & & & & & & & & $\bar{A}$ & $\mathrm{~A}$ & $\bar{A}$ & $\mathrm{~A}$ & $\mathrm{~A}$ & A & $\mathrm{A}$ & $\mathrm{A}$ & $\bar{A}$ & $\mathrm{~A}$ & $\mathrm{~A}$ \\
\hline 10 & $\overline{F G}$ & & & & & & & & & & & $\mathrm{~V}$ & $\mathrm{~V}$ & $\mathrm{~V}$ & $\mathrm{~V}$ & $\mathrm{~V}$ & $\mathrm{~V}$ & $\mathrm{~V}$ & $\mathrm{~V}$ & $\mathrm{~V}$ & $\mathrm{~V}$ \\
\hline 11 & LoR & & & & & & & & & & & & $\mathrm{V}$ & $\mathrm{V}$ & $\mathrm{V}$ & $\mathrm{V}$ & $\mathrm{V}$ & $\mathrm{V}$ & $\mathrm{V}$ & $\mathrm{V}$ & $\mathrm{V}$ \\
\hline 12 & $\mathrm{CP}$ & & & & & & & & & & & & & A & A & A & A & $\mathrm{A}$ & A & A & A \\
\hline 13 & MSM & & & & & & & & & & & & & & $\mathrm{A}$ & $\mathrm{A}$ & $\mathrm{A}$ & $\mathrm{A}$ & A & $\mathrm{A}$ & $\mathrm{A}$ \\
\hline 14 & AoF & & & & & & & & & & & & & & & V & $\mathrm{V}$ & $\mathrm{V}$ & V & $\mathrm{V}$ & $\mathrm{V}$ \\
\hline 15 & PL & & & & & & & & & & & & & & & & $\mathrm{V}$ & $\mathrm{V}$ & V & $\mathrm{V}$ & V \\
\hline 16 & LAR & & & & & & & & & & & & & & & & & $X$ & $\mathrm{X}$ & $X$ & $X$ \\
\hline 17 & CIA & & & & & & & & & & & & & & & & & & V & $\mathrm{V}$ & $\mathrm{V}$ \\
\hline 18 & UE & & & & & & & & & & & & & & & & & & & $\mathrm{A}$ & A \\
\hline 19 & MP & & & & & & & & & & & & & & & & & & & & $\mathrm{A}$ \\
\hline 20 & CMS & & & & & & & & & & & & & & & & & & & & \\
\hline
\end{tabular}

Fig 1: SSIM matrix for pair wise relationship amongst barriers

\begin{tabular}{|c|c|c|c|c|c|c|c|c|c|c|c|c|c|c|c|c|c|c|c|c|c|}
\hline & Barriers & 1 & 2 & 3 & 4 & 5 & 6 & 7 & 8 & 9 & 10 & 11 & 12 & 13 & 14 & 15 & 16 & 17 & 18 & 19 & 20 \\
\hline & & 3Ls & PIR & EUIS & $\overline{\mathrm{EHH}}$ & SCE & IRDE & AWF & HRP & ITG & FG & LoR & $\mathrm{CP}$ & MSMC & AoF & PL & LARP & CIA & UE & MP & CMS \\
\hline 1 & 3Ls & 1 & 1 & 1 & 1 & 1 & 1 & 1 & 1 & 1 & 1 & 1 & 1 & 1 & 1 & 1 & 1 & 1 & 1 & 1 & 1 \\
\hline 2 & PIR & 0 & 1 & 1 & 1 & 1 & 1 & 1 & 1 & 1 & 1 & 1 & 1 & 1 & 1 & 1 & 1 & 1 & 1 & 1 & 1 \\
\hline 3 & EUIS & 0 & 0 & 1 & 1 & 1 & 1 & 1 & 1 & 1 & 1 & 1 & 1 & 1 & 1 & 1 & 1 & 1 & 1 & 1 & 1 \\
\hline 4 & EHH & 0 & 0 & 0 & 1 & 1 & 0 & 0 & 0 & 0 & 0 & 0 & 0 & 0 & 0 & 0 & 0 & 0 & 0 & 0 & 0 \\
\hline 5 & $\overline{S C E}$ & 0 & 0 & 0 & 0 & 1 & 0 & 0 & 0 & 0 & 0 & 0 & 0 & 0 & 0 & 0 & 0 & 0 & 0 & 0 & 0 \\
\hline 6 & IRDE & 0 & 0 & 0 & 1 & 1 & 1 & 1 & 1 & 1 & 1 & 1 & 1 & 1 & 1 & 1 & 1 & 1 & 1 & 1 & 1 \\
\hline 7 & AWF & 0 & 0 & 0 & 1 & 1 & 0 & 1 & 1 & 1 & 1 & 1 & 1 & 1 & 1 & 1 & 1 & 1 & 1 & 1 & 1 \\
\hline 8 & HRP & 0 & 0 & 0 & 1 & 1 & 0 & 0 & 1 & 0 & 0 & 0 & 0 & 0 & 0 & 0 & 0 & 0 & 0 & 0 & 0 \\
\hline 9 & ITG & 0 & 0 & 0 & 1 & 1 & 0 & 0 & 1 & 1 & 0 & 0 & 0 & 0 & 0 & 0 & 0 & 0 & 0 & 0 & 0 \\
\hline 10 & FG & 0 & 0 & 0 & 1 & 1 & 0 & 0 & 1 & 1 & 1 & 1 & 1 & 1 & 1 & 1 & 1 & 1 & 1 & 1 & 1 \\
\hline 11 & LoR & 0 & 0 & 0 & 1 & 1 & 0 & 0 & 1 & 1 & 0 & 1 & 1 & 1 & 1 & 1 & 1 & 1 & 1 & 1 & 1 \\
\hline 12 & $\mathrm{CP}$ & 0 & 0 & 0 & 1 & 1 & 0 & 0 & 1 & 1 & 0 & 0 & 1 & 0 & 0 & 0 & 0 & 0 & 0 & 0 & 0 \\
\hline 13 & MSMC & 0 & 0 & 0 & 1 & 1 & 0 & 0 & 1 & 1 & 0 & 0 & 1 & 1 & 0 & 0 & 0 & 0 & 0 & 0 & 0 \\
\hline 14 & AoF & 0 & 0 & 0 & 1 & 1 & 0 & 0 & 1 & 1 & 0 & 0 & 1 & 1 & 1 & 1 & 1 & 1 & 1 & 1 & 1 \\
\hline 15 & PL & 0 & 0 & 0 & 1 & 1 & 0 & 0 & 1 & 1 & 0 & 0 & 1 & 1 & 0 & 1 & 1 & 1 & 1 & 1 & 1 \\
\hline 16 & LARP & 0 & 0 & 0 & 1 & 1 & 0 & 0 & 1 & 1 & 0 & 0 & 1 & 1 & 0 & 0 & 1 & 1 & 1 & 1 & 1 \\
\hline 17 & CIA & 0 & 0 & 0 & 1 & 1 & 0 & 0 & 1 & 1 & 0 & 0 & 1 & 1 & 0 & 0 & 1 & 1 & 1 & 1 & 1 \\
\hline
\end{tabular}




\begin{tabular}{|c|c|c|c|c|c|c|c|c|c|c|c|c|c|c|c|c|c|c|c|c|c|}
\hline 18 & UE & 0 & 0 & 0 & 1 & 1 & 0 & 0 & 1 & 1 & 0 & 0 & 1 & 1 & 0 & 0 & 1 & 0 & 1 & 0 & 0 \\
\hline 19 & MP & 0 & 0 & 0 & 1 & 1 & 0 & 0 & 1 & 1 & 0 & 0 & 1 & 1 & 0 & 0 & 1 & 0 & 1 & 1 & 0 \\
\hline 20 & CMS & 0 & 0 & 0 & 1 & 1 & 0 & 0 & 1 & 1 & 0 & 0 & 1 & 1 & 0 & 0 & 1 & 0 & 1 & 1 & 1 \\
\hline
\end{tabular}

Fig 2: Initial reachability matrix

\begin{tabular}{|c|c|c|c|c|c|c|c|c|c|c|c|c|c|c|c|c|c|c|c|c|c|}
\hline $\begin{array}{l}\mathrm{S} . \\
\mathrm{N}\end{array}$ & $\begin{array}{c}\text { Barri } \\
\text { ers }\end{array}$ & 1 & 2 & 3 & 4 & 5 & 6 & 7 & 8 & 9 & $\begin{array}{l}1 \\
0\end{array}$ & 11 & $\begin{array}{l}1 \\
2\end{array}$ & 13 & 14 & $\begin{array}{l}1 \\
5\end{array}$ & 16 & 17 & $\begin{array}{l}1 \\
8\end{array}$ & 19 & 20 \\
\hline & & $\begin{array}{c}3 \\
\text { Ls }\end{array}$ & $\begin{array}{l}\mathrm{PI} \\
\mathrm{R}\end{array}$ & $\begin{array}{l}\text { EU } \\
\text { IS }\end{array}$ & $\begin{array}{c}\mathrm{EH} \\
\mathrm{H}\end{array}$ & $\begin{array}{c}\text { SC } \\
\text { E }\end{array}$ & $\begin{array}{l}\text { IR } \\
\text { DE }\end{array}$ & $\begin{array}{c}\text { A } \\
\text { WF }\end{array}$ & $\begin{array}{c}\mathrm{H} \\
\mathrm{RP}\end{array}$ & $\begin{array}{l}\text { IT } \\
\text { G }\end{array}$ & $\begin{array}{l}F \\
G\end{array}$ & $\begin{array}{l}\text { Lo } \\
\text { R }\end{array}$ & $\begin{array}{l}\mathrm{C} \\
\mathrm{P}\end{array}$ & $\begin{array}{l}\text { MS } \\
\text { MC }\end{array}$ & $\begin{array}{c}\text { Ao } \\
\mathrm{F}\end{array}$ & $\begin{array}{l}\mathrm{P} \\
\mathrm{L}\end{array}$ & $\begin{array}{l}\text { LA } \\
\text { RP }\end{array}$ & $\begin{array}{c}\text { CI } \\
\text { A }\end{array}$ & $\begin{array}{l}\mathrm{U} \\
\mathrm{E}\end{array}$ & $\begin{array}{c}\mathrm{M} \\
\mathrm{P}\end{array}$ & $\begin{array}{c}\text { C } \\
\text { MS }\end{array}$ \\
\hline 1 & $3 \mathrm{Ls}$ & 1 & 1 & 1 & 1 & 1 & 1 & 1 & 1 & 1 & 1 & 1 & 1 & 1 & 1 & 1 & 1 & 1 & 1 & 1 & 1 \\
\hline 2 & PIR & 0 & 1 & 1 & 1 & 1 & 1 & 1 & 1 & 1 & 1 & 1 & 1 & 1 & 1 & 1 & 1 & 1 & 1 & 1 & 1 \\
\hline 3 & EUIS & 0 & 0 & 1 & 1 & 1 & 1 & 1 & 1 & 1 & 1 & 1 & 1 & 1 & 1 & 1 & 1 & 1 & 1 & 1 & 1 \\
\hline 4 & EHH & 0 & 0 & 0 & 1 & 1 & 0 & 0 & 0 & 0 & 0 & 0 & 0 & 0 & 0 & 0 & 0 & 0 & 0 & 0 & 0 \\
\hline 5 & SCE & 0 & 0 & 0 & 0 & 1 & 0 & 0 & 0 & 0 & 0 & 0 & 0 & 0 & 0 & 0 & 0 & 0 & 0 & 0 & 0 \\
\hline 6 & $\begin{array}{c}\text { IRD } \\
\mathrm{E}\end{array}$ & 0 & 0 & 0 & 1 & 1 & 1 & 1 & 1 & 1 & 1 & 1 & 1 & 1 & 1 & 1 & 1 & 1 & 1 & 1 & 1 \\
\hline 7 & AWF & 0 & 0 & 0 & 1 & 1 & 0 & 1 & 1 & 1 & 1 & 1 & 1 & 1 & 1 & 1 & 1 & 1 & 1 & 1 & 1 \\
\hline 8 & HRP & 0 & 0 & 0 & 1 & 1 & 0 & 0 & 1 & 0 & 0 & 0 & 0 & 0 & 0 & 0 & 0 & 0 & 0 & 0 & 0 \\
\hline 9 & ITG & 0 & 0 & 0 & 1 & 1 & 0 & 0 & 1 & 1 & 0 & 0 & 0 & 0 & 0 & 0 & 0 & 0 & 0 & 0 & 0 \\
\hline 10 & FG & 0 & 0 & 0 & 1 & 1 & 0 & 0 & 1 & 1 & 1 & 1 & 1 & 1 & 1 & 1 & 1 & 1 & 1 & 1 & 1 \\
\hline 11 & LoR & 0 & 0 & 0 & 1 & 1 & 0 & 0 & 1 & 1 & 0 & 1 & 1 & 1 & 1 & 1 & 1 & 1 & 1 & 1 & 1 \\
\hline 12 & $\mathrm{CP}$ & 0 & 0 & 0 & 1 & 1 & 0 & 0 & 1 & 1 & 0 & 0 & 1 & 0 & 0 & 0 & 0 & 0 & 0 & 0 & 0 \\
\hline 13 & $\begin{array}{l}\text { MS } \\
\text { MC }\end{array}$ & 0 & 0 & 0 & 1 & 1 & 0 & 0 & 1 & 1 & 0 & 0 & 1 & 1 & 0 & 0 & 0 & 0 & 0 & 0 & 0 \\
\hline 14 & AoF & 0 & 0 & 0 & 1 & 1 & 0 & 0 & 1 & 1 & 0 & 0 & 1 & 1 & 1 & 1 & 1 & 1 & 1 & 1 & 1 \\
\hline 15 & PL & 0 & 0 & 0 & 1 & 1 & 0 & 0 & 1 & 1 & 0 & 0 & 1 & 1 & 0 & 1 & 1 & 1 & 1 & 1 & 1 \\
\hline 16 & LAR & 0 & 0 & 0 & 1 & 1 & 0 & 0 & 1 & 1 & 0 & 0 & 1 & 1 & 0 & 0 & 1 & 1 & 1 & 1 & 1 \\
\hline 17 & CIA & 0 & 0 & 0 & 1 & 1 & 0 & 0 & 1 & 1 & 0 & 0 & 1 & 1 & 0 & 0 & 1 & 1 & 1 & 1 & 1 \\
\hline 18 & UE & 0 & 0 & 0 & 1 & 1 & 0 & 0 & 1 & 1 & 0 & 0 & 1 & 1 & 0 & 0 & 1 & 0 & 1 & 0 & 0 \\
\hline 19 & MP & 0 & 0 & 0 & 1 & 1 & 0 & 0 & 1 & 1 & 0 & 0 & 1 & 1 & 0 & 0 & 1 & 0 & 1 & 1 & 0 \\
\hline 20 & CMS & 0 & 0 & 0 & 1 & 1 & 0 & 0 & 1 & 1 & 0 & 0 & 1 & 1 & 0 & 0 & 1 & 0 & 1 & 1 & 1 \\
\hline
\end{tabular}

Fig 3 : Final reachability matrix

\begin{tabular}{|c|c|c|c|c|c|c|c|c|c|}
\hline S. No. & Barriers & 1 & 2 & 3 & 4 & 5 & 6 & 7 & 8 \\
\hline & & & & & & & & & \\
\hline 1 & CSP & & V & V & V & V & X & V & V \\
\hline 2 & GoR & & & A & A & A & A & A & A \\
\hline 3 & RE & & & & V & V & V & V & V \\
\hline 4 & IRR & & & & & A & A & A & A \\
\hline 5 & PA & & & & & & A & V & X \\
\hline 6 & AIC & & & & & & & V & V \\
\hline 7 & BFM & & & & & & & & V \\
\hline 8 & TEM & & & & & & & & \\
\hline
\end{tabular}

Fig 4 : SSIM for success factors 


\begin{tabular}{|c|c|c|c|c|c|c|c|c|c|}
\hline S. No. & Barriers & 1 & 2 & 3 & 4 & 5 & 6 & 7 & 8 \\
\hline & & CSP & GoR & RE & IRR & PA & AIC & BFM & TEM \\
\hline 1 & CSP & 1 & 1 & 1 & 1 & 1 & 1 & 1 & 1 \\
\hline 2 & GoR & 0 & 1 & 0 & 0 & 0 & 0 & 0 & 0 \\
\hline 3 & RE & 0 & 1 & 1 & 1 & 1 & 1 & 1 & 1 \\
\hline 4 & IRR & 0 & 1 & 0 & 1 & 0 & 0 & 0 & 0 \\
\hline 5 & PA & 0 & 1 & 0 & 1 & 1 & 0 & 1 & 1 \\
\hline 6 & AIC & 1 & 1 & 0 & 1 & 1 & 1 & 1 & 1 \\
\hline 7 & BFM & 0 & 1 & 0 & 1 & 0 & 0 & 1 & 1 \\
\hline 8 & TEM & 0 & 1 & 0 & 1 & 1 & 0 & 0 & 1 \\
\hline
\end{tabular}

Fig 5: Initial reachability matrix for success factors

\begin{tabular}{|c|c|c|c|c|c|c|c|c|c|c|}
\hline $\begin{array}{c}\text { S. } \\
\text { No. }\end{array}$ & Barriers & 1 & 2 & 3 & 4 & 5 & 6 & 7 & 8 & D.P \\
\hline & & CSP & GoR & RE & IRR & PA & AIC & BFM & TEM & \\
\hline 1 & CSP & 1 & 1 & 1 & 1 & 1 & 1 & 1 & 1 & 8 \\
\hline 2 & GoR & 0 & 1 & 0 & 0 & 0 & 0 & 0 & 0 & 1 \\
\hline 3 & RE & 0 & 1 & 1 & 1 & 1 & 1 & 1 & 1 & 7 \\
\hline 4 & IRR & 0 & 1 & 0 & 1 & 1 & 1 & 1 & 1 & 6 \\
\hline 5 & PA & 0 & 1 & 0 & 1 & 1 & 0 & 1 & 1 & 5 \\
\hline 6 & AIC & 1 & 1 & 1 & 1 & 1 & 1 & 1 & 1 & 7 \\
\hline 7 & BFM & 0 & 1 & 0 & 1 & 0 & 0 & 1 & 1 & 4 \\
\hline 8 & TEM & 0 & 1 & 0 & 1 & 1 & 1 & 1 & 1 & 6 \\
\hline & De.P & 2 & 8 & 3 & 7 & 6 & 5 & 7 & 7 & \\
\hline
\end{tabular}

Fig 6 : Final reachability matrix for success factors

\subsection{Level Partition}

Table 4.3.1 Iteration 1

\begin{tabular}{|c|c|c|c|c|}
\hline S.no & $\begin{array}{c}\text { Reachability } \\
\text { set }\end{array}$ & $\begin{array}{c}\text { Antecedent } \\
\text { set }\end{array}$ & $\begin{array}{c}\text { Intersection } \\
\text { set }\end{array}$ & Level \\
\hline 1 & 2 & $1,2,3,4,5,6,7,8$ & 2 & I \\
\hline 2 & $2,4,7,8$ & $1,3,4,5,6,7,8$ & $4,7,8$ & II \\
\hline 3 & $2,4,5,7,8$ & $1,3,4,5,6,8$ & $4,5,8$ & III \\
\hline 4 & $2,4,5,6,7,8$ & $1,3,4,6,8$ & $4,6,8$ & IV \\
\hline 5 & $2,3,4,5,6,7,8$ & $1,3,6$ & 3,6 & V \\
\hline 6 & $1,2,3,4,5,6,7,8$ & 1,6 & 1,6 & VI \\
\hline
\end{tabular}

\section{HYBRID APPROACHES TO e- WASTE MANAGEMENT}

Inclusive and formal-informal hybrid models for cleaner ewaste can add significant value and there are efficiency gains in models that integrate small-scale and informal enterprises.

\section{LITERARY OBSERVATIONS AND RECOMMENDATIONS}

- The potential impacts of 'modernisation' of $\boldsymbol{e}$-waste management on the poor and vulnerable are significant. As more developing countries start to grapple with new domestic e-waste streams, exclusionary policies of formalisation and privatisation of waste will impact millions of lowincome livelihoods.

- Hybrid models must recognise and avoid many potential risks - such as intensifying vulnerabilities, power imbalances in the design of operating models, diverging interests leading to exploitation, and undermining of informal worker organisations[21].

- Effective interventions addressing livelihoods, health and the environment together must also confront the realities of e-waste pricing within established informal markets.

- Adhering to technical standards has a bearing on costs, especially in the distinction between the informal and formal sector. Therefore, it is essential for the regulator to establish clear standards for collection, transport, dismantling, treatment and disposal. There are several international standards such as CENELEC ${ }^{a}$ and R2 ${ }^{b}$

- ILO recommends strengthening and/or formalising the collection phases of informal e-waste recovery. This option requires the least capital investment and could bridge both informal (collectors) and formal (recycling companies) sectors and use their 
respective skills to build more inclusive and sustainable $e$-waste chains [22-23].

\section{ACKNOWLEDGMENTS}

Our thanks to the anonymous reviewers whose comments have helped us in improving the manuscript . Co-author Remica Aggarwal also pay her sincere regards to Prof. S.P Singh of DMS , IIT Delhi for disseminating the knowledge about ISM methodology. This manuscript is a tribute to the Delhi district Seelampur which is considered as a major centre for $e$-waste in Delhi .

\section{REFERENCES}

[1] ASSOCHAM (2014) Over 4.5 lakhs child labours engaged in various $e$-waste activities in India; India's capital produces 67,000 MT of electronic waste a year. Press release. http://bit.ly/1OTpklI .

[2] Carisma, B. 2009. Drivers of and barriers to E-waste management in the Philippines. https://www.researchgate.net/publication/40223372_Driv ers_of_and_barriers_to_Ewaste_management_in_the_Philippines

[3] Kumar, A. , Dixit , G. 2018. An analysis of barriers affecting the implementation of e-waste management practices in India: A novel ISM-DEMATEL approach , DOI: 10.1016/j.spc.2018.01.002

[4] Sthiannopkao, S and Wong, M. 2012. Handling e-waste in developed and developing countries: Initiatives, practices, and consequences. Science of the Total Environment 463: 1,147-1,153

[5] Balde, CP, Kuehr, R, Blumenthal, K, Fondeur Gill, S, Kern, M, Micheli, P, Magpantay, E, Huisman, J 2015. The global e-waste monitor - 2014. United Nations University, IAS-SCYCLE, Bonn, Germany.

[6] Borthakur, A and Singh, P. 2012. Electronic waste in India: Problems and policies. International Journal of Environmental Sciences 3(1), 353-362.

[7] Brigden, K, Labunska, I, Santillo, D and Allsopp, M. 2005. Recycling of electronic wastes in China and India: Workplace and environmental contamination. Greenpeace International.

[8] Chaturvedi, A, Khattar, V, Kaur, J and Arora, R 2007. Ewaste assessment in India - specific focus on Delhi. MAIT and GTZ

[9] Chaturvedi, B and Bhardwak, S. 2013. Learning to re-ecycle: What working with e-waste has taught us. Chintan Environmental Research and Action Group, New Delhi.

[10] Chaturvedi, A, Arora, R and Ahmed, S. 2010. Mainstreaming the informal sector in e-waste management. GTZ Advisory Services in Environmental Management. Urban, Industrial and Hospital Waste Management Conference, Ahmedabad, 7-8 May 2010. http://tinyurl.com/chaturvedi-etal-weee-2010
[11] Chaturvedi, A, Arora, R, Killguss, U (2011) E-waste recycling in India: Bridging the formal-informal divide.

[12] Barba-Gutiérrez, Y, Adenso-Díaz, B, Hopp, M 2008. An analysis of some environmental consequences of European electrical and electronic waste regulation. Resources, Conservation and Recycling 52(3): 481-495.

[13] Adelphi, Toxics Link, \& Strategos Advisory. 2017. Building the link leveraging formal- informal partnerships in the Indian E-Waste sector. Bonn and Eschborn, Germany: GIZ.

[14] Gerdes, P and Gunsilius, E. 2010. The waste experts: Enabling conditions for informal sector integration in solid waste management: Lessons learned from Brazil, Egypt and India. GTZ.

[15] Wilson, E. 2015. Managing the emerging waste crisis in developing countries' large cities. IDS Policy Briefing, Institute of Development Studies, Brighton, UK.

[16] Eisinger, F, Chakrabarti, R, Krüger, C and Alexeew, J.2011. Carbon footprint of $e$-waste recycling scenarios in India. Adelphi, Berlin.

[17] Schluep, M, Mathias Schluepa Hageluekenb, C, Kuehrc, R, Magalinic, F, Maurerc, C, Meskersb, C, Muellera, E and Wangc, F. (2009) Sustainable innovation and technology transfer industrial sector studies: Recyclingfrom $e$-waste to resources. UNEP and United Nations University, Bonn, Germany.

[18] Sepúlveda, A, Schleup, M, Renaud, FG, Streicher, M, Kuehr, R, Hagelüken, C and Gerecke, AC. 2010. A review of the environmental fate and effects of hazardous substances released from electrical and electronic equipments during recycling: examples from China and India. Environmental Impact Assessment Review 30(1), $28-41$

[19] Shinkuma, T and Nguyen, T.M.H. 2009. The flow of $e$ waste material in the Asian region and a reconsideration of international trade policies on e-waste. Environmental Impact Assessment Review 29(1), 25-31.

[20] Warfield, J. N. 1974. Developing interconnection matrices in structural modeling. IEEE Transactions on System, Man, and Cybernetics, SMC-4 (1), 81-87.

[21] Meagher, K. 2013. Unlocking the informal economy: A literature review on linkages between formal and informal economies in developing countries. WIEGO Working Paper 27.

[22] ILO 2014. Tackling informality in $e$-waste management The potential of cooperative enterprises. International Labour Organization, Sectoral Activities Department, Cooperatives Unit, Geneva.

[23] Sthiannopkao, S and Wong, M. 2012. Handling e-waste in developed and developing countries: Initiatives, practices, and consequences. Science of the Total Environment 463: 1,147-1,153. 